\title{
Research Article Steady Heat Transfer through a Two-Dimensional Rectangular Straight Fin
}

\author{
Raseelo J. Moitsheki and Atish Rowjee \\ Center for Differential Equations, Continuum Mechanics and Applications, School of Computational and \\ Applied Mathematics, University of the Witwatersrand, Private Bag 3, Wits, \\ Johannesburg 2050, South Africa
}

Correspondence should be addressed to Raseelo J. Moitsheki, raseelo.moitsheki@wits.ac.za

Received 9 December 2010; Accepted 14 February 2011

Academic Editor: Reza Jazar

Copyright (C) 2011 R. J. Moitsheki and A. Rowjee. This is an open access article distributed under the Creative Commons Attribution License, which permits unrestricted use, distribution, and reproduction in any medium, provided the original work is properly cited.

\begin{abstract}
Exact solutions for models describing heat transfer in a two-dimensional rectangular fin are constructed. Thermal conductivity, internal energy generation function, and heat transfer coefficient are assumed to be dependent on temperature. We apply the Kirchoff transformation on the governing equation. Exact solutions satisfying the realistic boundary conditions are constructed for the resulting linear equation. Symmetry analysis is carried out to classify the internal heat generation function, and some reductions are performed. Furthermore, the effects of physical parameters such as extension factor (the purely geometric fin parameter) and Biot number on temperature are analyzed. Heat flux and fin efficiency are studied.
\end{abstract}

\section{Introduction}

Fins are extended surfaces used to increase the heat transfer rate between a hot body and its surroundings. There are a variety of uses such as in air conditioning systems, compressors, and cooling of electronic components. The theory on heat transfer in extended surface may be found in texts such as $[1,2]$. Few exact solutions exist even for the one dimensional fin problem with constant thermal conductivity and heat transfer coefficient [3]. Series solutions for one-dimensional fin problem with constant heat transfer coefficient and temperaturedependent thermal conductivity are given in [4]. Furthermore, analytical and exact solutions for one dimensional fins models with temperature-dependent thermal conductivity and heat transfer coefficient were obtained for example, in [5-7]. A compendium of heat transfer in all types of one dimensional fins is given in [8]. Exact steady-state solutions exist for twodimensional models with constant thermal conductivity and heat transfer coefficient, with no internal heat generation [9-15], and with internal heat generation function depending on a spatial variable $[16,17]$. Solutions for transient heat transfer in fins are constructed in [18]. 


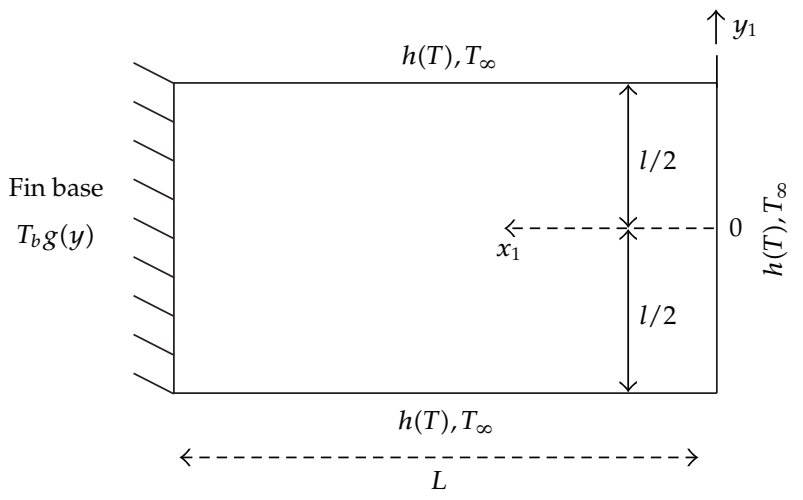

Figure 1: Schematic representation of a rectangular straight fin.

In this paper, we apply the Kirchoff transformation to the two-dimensional steadystate heat balance equation in Section 2. The resulting model contains the arbitrary source term. In Section 3, we construct exact solutions which satisfy the physical boundary conditions. Symmetry analysis is carried out for the single governing equation, wherein group classification is performed in Section 4. Heat transfer results including fin efficiency and heat flux are analyzed in Section 5. Lastly, we provide concluding remarks in Section 6.

\section{Mathematical Model}

We consider a two-dimensional rectangular fin of length $L$ as shown in Figure 1. The fin is mounted to a base surface of temperature $T_{b} g(y)$ and extended into its surrounding of temperature $T_{\infty}$. The heat flow is assumed to be symmetric along the line $y=0$. We assume that the heat transfer coefficient along the fin is nonuniform and temperature dependent. Also, the internal heat generation is nonzero and temperature dependent.

The two-dimensional heat balance equation is given by (see, e.g., $[16,17]$ )

$$
\frac{\partial}{\partial y_{1}}\left[K(T) \frac{\partial T}{\partial y_{1}}\right]+\frac{\partial}{\partial x_{1}}\left[K(T) \frac{\partial T}{\partial x_{1}}\right]=s(T)
$$

The imposed boundary conditions are

$$
\begin{gathered}
K(T) \frac{\partial T}{\partial x_{1}}=-H(T)\left[T\left(0, y_{1}\right)-T_{\infty}\right], \quad x_{1}=0 \\
T\left(L, y_{1}\right)=\left(T_{b}-T_{\infty}\right) g\left(y_{1}\right), \quad x_{1}=L \\
\frac{\partial T}{\partial y_{1}}=0, \quad y_{1}=0, \\
K(T) \frac{\partial T}{\partial y_{1}}=-H(T)\left[T\left(x_{1}, \frac{l}{2}\right)-T_{\infty}\right], \quad y_{1}=\frac{l}{2} .
\end{gathered}
$$


Here, $T$ is the dimensionless temperature, $T_{b}$ is the fin base temperature, $H$ is the heat transfer coefficient, $x_{1}$ is the longitudinal coordinate, $y_{1}$ is the transverse coordinate, $S$ is the internal heat generation function, and $K$ is the thermal conductivity. Several authors have considered the two-dimensional problem with $S=0$ and thermal conductivity being a constant (see, e.g., $[19,20]$ ) and the case $S=0$ with a temperature-dependent thermal conductivity [21].

Introducing the dimensionless variables

$$
\begin{gathered}
\theta=\frac{T-T_{\infty}}{T_{b}-T_{\infty}}, \quad x=\frac{x_{1}}{L}, \quad y=\frac{y_{1}}{l / 2}, \quad k(\theta)=\frac{K(T)}{K_{a}}, \\
h(\theta)=\frac{H(T)}{h_{b}}, \quad E^{2}=\left(\frac{L}{l / 2}\right)^{2}, \quad S(\theta)=\frac{s(T)(l / 2)^{2}}{K_{a}\left(T_{b}-T_{\infty}\right)},
\end{gathered}
$$

we obtain

$$
E^{2} \frac{\partial}{\partial y}\left[k(\theta) \frac{\partial \theta}{\partial y}\right]+\frac{\partial}{\partial x}\left[k(\theta) \frac{\partial \theta}{\partial x}\right]=E^{2} S(\theta)
$$

The corresponding dimensionless boundary conditions are

$$
\begin{gathered}
k(\theta) \frac{\partial \theta}{\partial x}=-\mathrm{Bi}_{\phi} h(\theta) \theta, \quad x=0, \\
\theta(1, y)=g(y), \quad x=1, \\
\frac{\partial \theta}{\partial y}=0, \quad y=0, \\
k(\theta) \frac{\partial \theta}{\partial y}=-\mathrm{Bi}_{\psi} h(\theta) \theta, \quad y=1,
\end{gathered}
$$

where $E$ is the fin extension factor (purely geometric parameter), and $\mathrm{Bi}_{\phi}=h_{b} L / K_{a}$ and $\mathrm{Bi}_{\psi}=h_{b}(l / 2) / K_{a}$ are the Biot numbers. $E$ is the reciprocal to aspect ratio (see, e.g., [16]). $h_{b}$ and $k_{a}$ are the heat transfer at the base and thermal conductivity of the fin at the ambient temperature, respectively.

\section{Exact Solutions}

In this section, we construct exact solutions for the boundary value problem (BVP) (2.4)(2.5). The problem is simplified by the introduction of the Kirchoff transformation. The Kirchoff's transformation (see, e.g., [21])

$$
\omega(x, y)=\int_{\theta_{0}}^{\theta} k\left(\theta^{*}\right) d \theta^{*}
$$


with $\theta_{0}$ being an arbitrary constant, reduces the BVP (2.4)-(2.5) to

$$
E^{2} \frac{\partial^{2} \omega}{\partial y^{2}}+\frac{\partial^{2} \omega}{\partial x^{2}}=E^{2} S(\omega)
$$

subject to the conditions

$$
\begin{gathered}
\frac{\partial \omega}{\partial x}+\mathrm{Bi}_{\phi} \omega=0, \quad x=0, \\
\frac{\partial \omega}{\partial y}+\mathrm{Bi}_{\psi} \omega=0, \quad y=1, \\
\frac{\partial \omega}{\partial y}=0, \quad y=0, \\
\omega(1, y)=G(y) .
\end{gathered}
$$

We consider two cases of the thermal conductivity. Note that in (3.3) and (3.4), we required the product of the heat transfer coefficient and temperature to match the integral of thermal conductivity. In fact, in a one-dimensional case, $h$ must be the differential consequence of $k$ (see, e.g., [7]). $G(y)$ is quadratic in $g(y)$ when $K$ is linear, and $G$ is given by the power law $g(y)^{n+1}$ when $K$ is nonlinear.

\subsection{Case 1. Linear Thermal Conductivity}

Thermal conductivity is assumed to be a linear function of temperature for many engineering applications [3]. We assume thermal conductivity to be linear function of temperature (see also $[21,22])$

$$
K(T)=k_{a}\left(1+\beta\left(T-T_{\infty}\right)\right) .
$$

$\beta$ is the parameter that describes temperature dependency $[3,4]$. In dimensionless variables, $k(\theta)=1+B \theta$ where $B=\beta\left(T_{b}-T_{\infty}\right)$. This case of $k$ requires $h(\theta)=(1+(B / 2) \theta)-\left(\theta_{0}+\right.$ $\left.(B / 2) \theta_{0}^{2}\right) \theta^{-1}$, so that BVP (3.2)-(3.6) hold. Note that $\theta=0$ renders heat transfer coefficient to be singular. However, one can remove singularity by choosing, without loss of generality, $\theta_{0}=0$. Assuming the internal heat generation to be linearly dependent on temperature, then the governing equation becomes the modified Helmholtz type equation

$$
E^{2} \frac{\partial^{2} \omega}{\partial y^{2}}+\frac{\partial^{2} \omega}{\partial x^{2}}=E^{2} \omega
$$

We seek exact solutions of (3.8) subject to (3.3)-(3.6). Using method of separation of variables, we obtain the nontrivial exact solutions for two cases of the separation constant $\sigma$. Note that $\sigma=0$ leads to trivial solutions. 
Table 1: Extra point symmetries admitted by (3.2).

\begin{tabular}{lc}
\hline$S(\omega)$ & Symmetries \\
\hline$\left(\alpha_{1}+\alpha_{2} \omega\right)^{m} ; \alpha_{1}, \alpha_{2} \in \Re, m \neq 0,1$ & $X_{4}=-\left(2\left(\alpha_{1}+\alpha_{2} \omega\right) / \alpha_{2}(m-1)\right)(\partial / \partial \omega)+y(\partial / \partial y)+x(\partial / \partial x)$ \\
$\omega^{m}, m \neq 0,1$ & $X_{4}=-(2 \omega /(m-1))(\partial / \partial \omega)+y(\partial / \partial y)+x(\partial / \partial x)$ \\
$\omega$ & $X_{4}=\omega(\partial / \partial \omega), X_{5}=P(x, y)(\partial / \partial \omega)$ \\
\hline
\end{tabular}

3.1.1. $\sigma<0$; say $\sigma=-\lambda^{2}, \lambda>0$

$$
\omega(x, y)=r \cosh (\lambda y)\left[\cos \left(E \sqrt{\lambda^{2}-1} x\right)-\frac{\mathrm{Bi}_{\phi}}{E \sqrt{\lambda^{2}-1}} \sin \left(E \sqrt{\lambda^{2}-1} x\right)\right], \quad \lambda^{2} \neq 1
$$

where $\gamma$ is an arbitrary constant and $\lambda$ satisfies $\lambda \tanh (\lambda)=-\mathrm{Bi}_{\psi}$. Also,

$$
\omega(x, y)=r \cosh (y)\left(1+\mathrm{Bi}_{\phi} x\right), \quad \lambda^{2}=1
$$

3.1.2. $\sigma>0$; say $\sigma=\lambda^{2}$

$$
\omega(x, y)=\sum_{n=1}^{\infty} k_{n} \cos \left(\lambda_{n} y\right)\left[\cosh \left(E \sqrt{\lambda_{n}^{2}+1} x\right)-\frac{\mathrm{Bi}_{\phi}}{E \sqrt{\lambda_{n}^{2}+1}} \sinh \left(E \sqrt{\lambda_{n}^{2}+1} x\right)\right]
$$

where $\lambda_{n}$ is satisfied by

$$
\begin{gathered}
\lambda_{n} \tan \left(\lambda_{n}\right)=\mathrm{Bi}_{\psi} \\
k_{n}=2\left[\cosh \left(E \sqrt{\lambda_{n}^{2}+1}\right)-\frac{\mathrm{Bi}_{\phi}}{E \sqrt{\lambda_{n}^{2}+1}} \sinh \left(E \sqrt{\lambda_{n}^{2}+1}\right)\right]_{0}^{-1} \cos \left(\lambda_{n} y\right) G(y) d y .
\end{gathered}
$$

The temperature profile for solution (3.11) is given in Figures 2(a) and 2(b). In Figures 3(a) and $3(\mathrm{~b})$, we plot the temperature profile at the boundaries $y=1$ and $x=0$, respectively. For simplicity, we allowed $\mathrm{Bi}_{\phi}$ and $\mathrm{Bi}_{\psi}$ to be equal. We list the first five eigenvalues of (3.12) for various values of the Biot number in Table 2.

In terms of the original temperature variable, we obtain the exact solution

$$
\theta=\frac{-1 \pm \sqrt{1+2 B(\omega+\delta)}}{B}
$$

where $\delta=\theta_{0}+(B / 2) \theta_{0}^{2}$. 


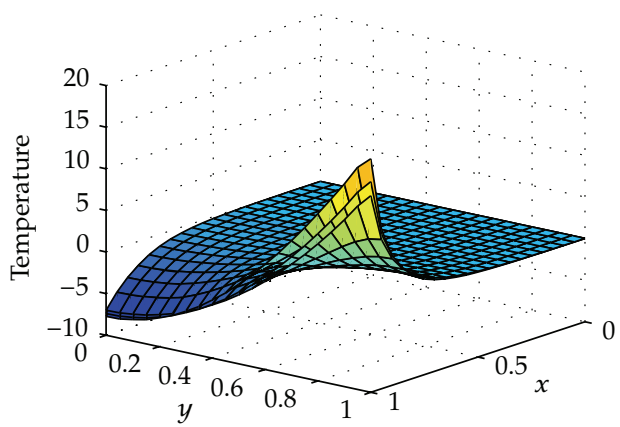

(a) Parameters used are $\mathrm{Bi}_{\phi}=\mathrm{Bi}_{\psi s}=0.01,0.5,2$ from bottom to top sheet, respectively. $E$ is fixed at 2

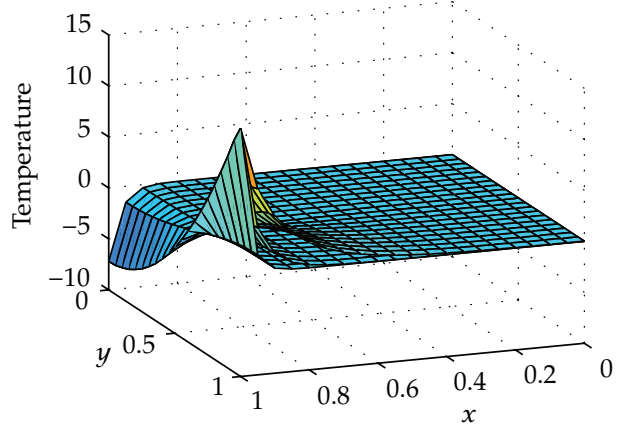

(b) Parameters used are $E=2,5,10$ from top to bottom sheet, respectively. Both $\mathrm{Bi}_{\phi}$ and $\mathrm{Bi}_{\psi}$ are fixed at 0.2

Figure 2: Temperature profile for the first twenty terms of the series given in (3.11).

Table 2: The first five eigenvalues of (3.12).

\begin{tabular}{lccccc}
\hline $\mathrm{Bi}_{\psi}$ & 0.2 & 0.4 & 0.6 & 0.8 & 1.0 \\
\hline$\lambda_{1}$ & 3.204 & 3.264 & 3.320 & 3.374 & 3.426 \\
$\lambda_{2}$ & 6.315 & 6.346 & 6.377 & 6.407 & 6.437 \\
$\lambda_{3}$ & 9.446 & 9.467 & 9.488 & 9.509 & 9.529 \\
$\lambda_{4}$ & 12.582 & 12.598 & 12.614 & 12.630 & 12.645 \\
$\lambda_{5}$ & 15.720 & 15.733 & 15.746 & 15.759 & 15.771 \\
\hline
\end{tabular}

\subsection{Case 2: Nonlinear Thermal Conductivity}

Assuming the power law temperature-dependent thermal conductivity

$$
K(T)=k_{a}\left(\frac{T-T_{\infty}}{T_{b}-T_{\infty}}\right)^{n}
$$

which is given in dimensionless variables as $k(\theta)=\theta^{n}$, requires

$$
h(\theta)=\frac{\theta^{n}}{n+1}-\frac{\theta_{0}^{n+1}}{(n+1) \theta}, \quad n \neq-1
$$

Again, one may, without loss of generality, assume, $\theta_{0}=0$. We obtain in terms of original variables the exact solution

$$
\begin{gathered}
\theta=[(n+1) \omega]^{1 /(n+1)}, \quad n \neq-1, \\
\theta=\exp (\omega), \quad n=-1,
\end{gathered}
$$

where $\omega$ is given in Sections 3.1.1 and 3.1.2. 


\section{Symmetry Analysis}

In this section, we analyze (3.2) with an arbitrary source term. We employ symmetry techniques. A symmetry group of a system of differential equations is a group of transformations mapping any arbitrary solution to another solution of the system. Such groups depend on continuous parameters. Given a continuous one-parameter symmetry group, in most practical cases, one may reduce the number of independent variables by one. The most familiar symmetry is the rotational symmetry that enables one to reduce the variables $(x, y)$ to a single radial variable $r$. Recent accounts on this theory may be found in many excellent texts such as those of [23, 24]. We use the methods in [24] (which exclude the use of equivalence transformations) to determine possible cases of $S$ for which extra symmetries are obtained.

In the initial symmetry analysis where $S$ is arbitrary, we obtain the translations of the spatial variables $x$ and $y$ and the rotational symmetry. Cases for which extra symmetries are obtained are listed in Table 1. Note that $S=0$ reduces (3.2) to the steady two-dimensional thermal diffusion equation (the Laplace equation which has been already analyzed).

\subsection{Symmetry Reductions: Illustrative Example}

Given the internal heat generation term as the power law $s(w)=w^{m}, m \neq 1$ (as listed in Table 1), $X_{4}$ leads to the functional form

$$
\omega=x^{2 /(1-m)} F(\xi), \quad \xi=\frac{x}{y}
$$

where $F$ satisfies a nonlinear ordinary differential equation (ODE)

$$
\left(1-E^{2} \xi^{2}\right) F^{\prime \prime}+\left[2 E^{2} \xi+\left(\frac{4}{1-m}\right) \frac{1}{\xi}\right] F^{\prime}+\left(\frac{2}{1-m}\right)\left(\frac{2}{1-m}-1\right) \frac{1}{\xi} F-\left(\frac{E}{\xi}\right)^{2} F^{m}=0 .
$$

The ODE (4.2) is harder to solve exactly. Furthermore, the boundary conditions are not invariant under $X_{4}$. In fact, the BVP (2.4)-(2.5) is not invariant under all the admitted symmetries listed. One may seek numerical solutions when internal heat generation term is nonlinear. We omit numerical analysis in this paper. However, the obtained exact solutions in Section 3 may be used as benchmarks for the numerical schemes.

\section{Heat Transfer Results}

The number of eigenvalues required to calculate the temperature distribution, heat flux, and fin efficiency accurately depends on the Biot number $\mathrm{Bi}_{\psi}$. We observe in Table 2 below that Biot number is directly proportional to the eigenvalues. Similar results are obtained for heat transfer in orthotropic convective pin fin [14]. The expression for the temperature distribution is given explicitly in (3.9) and (3.11). However, in further analysis, we focus on solution (3.11). The temperature distribution depends on a number of variables including $\mathrm{Bi}_{\phi}, \mathrm{Bi}_{\psi}$, eigenvalues, and the arbitrary function of $y$ describing the temperature at the base of the fin. We are free to choose any function $G$. Note that temperature distribution is proportional to both $\mathrm{Bi}_{\phi}$ and $\mathrm{Bi}_{\psi}$. 


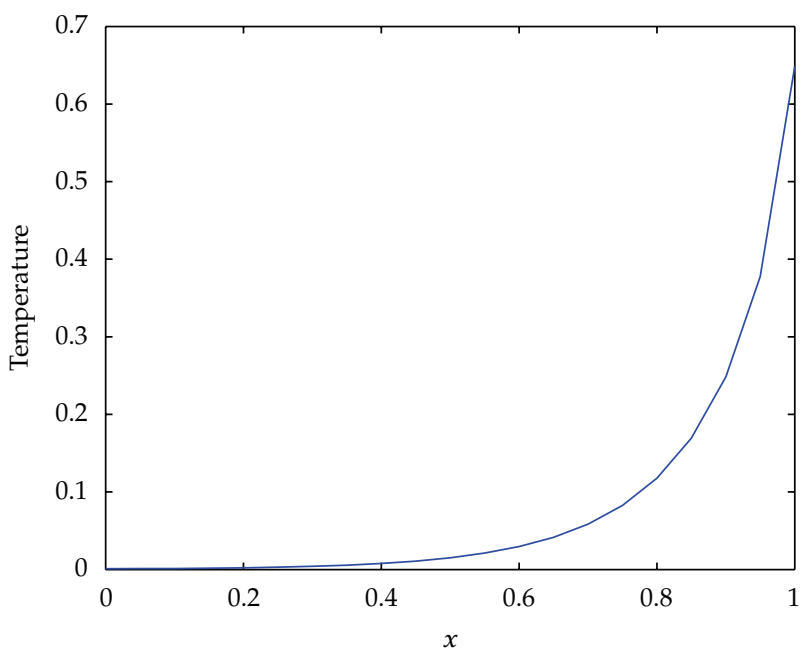

(a) Temperature profile at $y=1$. Parameters used are $\mathrm{Bi}_{\phi}=\mathrm{Bi}_{\psi}=0.02$, $E=2$

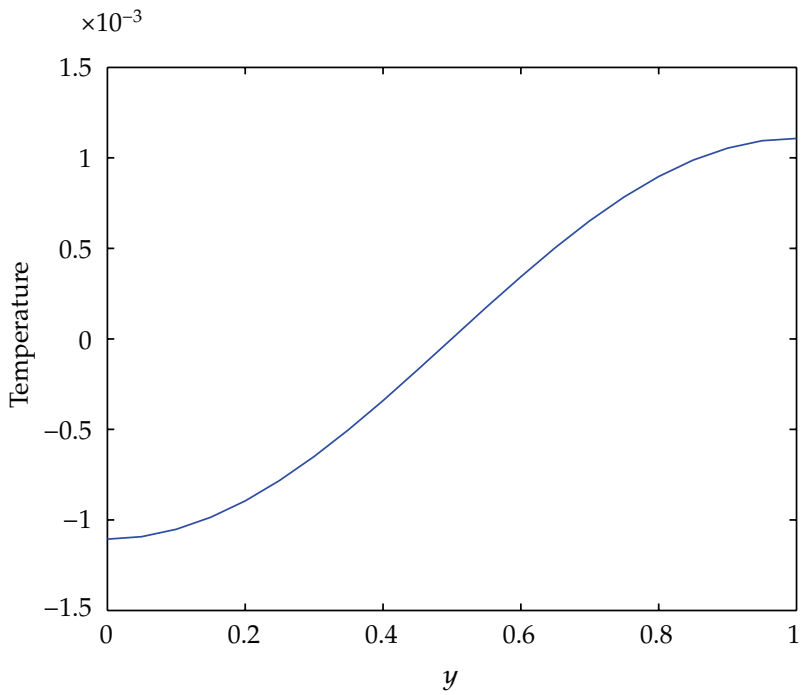

(b) Temperature profile at $x=0$. Parameters used are $\mathrm{Bi}_{\phi}=\mathrm{Bi}_{\psi}=0.02$, $E=2$

Figure 3: Graphical representation of temperature distribution at the fin boundaries.

We observe in Figure 2(a) that the Biot number is directly proportional to the temperature distribution in the fin. Also, in Figure 2(b), we notice that temperature decreases with the increase of the extension factor. Clearly, if the length of the fin is increased, temperature is at its lowest value, or increased width of the fin results in increased temperature distribution. Figures 3(a) and 3(b) depict the temperature distribution at the boundaries of the fin. We note that, in Figure 3(a), there is a significant reduction in temperature along the boundary and toward the tip of the fin (one may recall that we assumed that the fin is measured from the tip to the base). Figure 3(b) shows temperature variation at the tip of the fin. 


\subsection{Fin Efficiency and Heat Flux}

\subsubsection{Heat Flux}

The heat transfer from the fin base may be constructed by evaluating heat conduction rate at the base (see, e.g., [12])

$$
q_{b}=\left.\int_{0}^{\ell / 2} K(T) \frac{\partial T}{\partial x_{1}}\right|_{x_{1}=L} d y_{1}=\left.\frac{K_{a}\left(T_{b}-T_{\infty}\right) l / 2}{L} \int_{0}^{1} k(\theta) \frac{\partial \theta}{\partial x}\right|_{x=1} d y
$$

The dimensionless heat transfer rate from the base of the fin is defined by [12]

$$
Q=\frac{q_{b} L}{K_{a}\left(T_{b}-T_{\infty}\right) l / 2}=\left.\int_{0}^{1} k(\theta) \frac{\partial \theta}{\partial x}\right|_{x=1} d y
$$

\subsubsection{Fin Efficiency}

Fin efficiency (overall fin performance) is defined as the ratio of the actual heat transfer from the fin rate of heat that would be ideally transferred if the entire fin was at the temperature of the fin base [25]. The local fin efficiency is defined by [13]

$$
\eta=\frac{q_{b}}{Q_{i}}=\frac{\left.\left(\left(K_{a}\left(T_{b}-T_{\infty}\right) l / 2\right) / L\right) \int_{0}^{1} k(\theta)(\partial \theta / \partial x)\right|_{x=1} d y}{h_{b}\left(T_{b}-T_{\infty}\right) l / 2},
$$

or simply

$$
\eta=\left.\frac{1}{E \operatorname{Bi}_{\psi}} \int_{0}^{1} k(\theta) \frac{\partial \theta}{\partial x}\right|_{x=1} d y
$$

\subsubsection{Flux and Fin Efficiency Given (3.11)}

Given the solution (3.11), we obtain heat flux in terms of $w$,

$$
Q=\left.\int_{0}^{1} \frac{\partial w}{\partial x}\right|_{x=1} d y=\sum_{n=1}^{\infty} k_{n} \frac{\sin \left(\lambda_{n}\right)}{\lambda_{n}}\left[E \sqrt{\lambda_{n}^{2}+1} \sinh \left(E \sqrt{\lambda_{n}^{2}+1}\right)-B i_{\phi} \cosh \left(E \sqrt{\lambda_{n}^{2}+1}\right)\right]
$$

and fin efficiency

$$
\begin{aligned}
\eta & =\left.\frac{1}{E \mathrm{Bi}_{\psi}} \int_{0}^{1} \frac{\partial w}{\partial x}\right|_{x=1} d y \\
& =\frac{1}{E \mathrm{Bi}_{\psi}} \sum_{n=1}^{\infty} k_{n} \frac{\sin \left(\lambda_{n}\right)}{\lambda_{n}}\left[E \sqrt{\lambda_{n}^{2}+1} \sinh \left(E \sqrt{\lambda_{n}^{2}+1}\right)-\mathrm{Bi}_{\phi} \cosh \left(E \sqrt{\lambda_{n}^{2}+1}\right)\right] .
\end{aligned}
$$




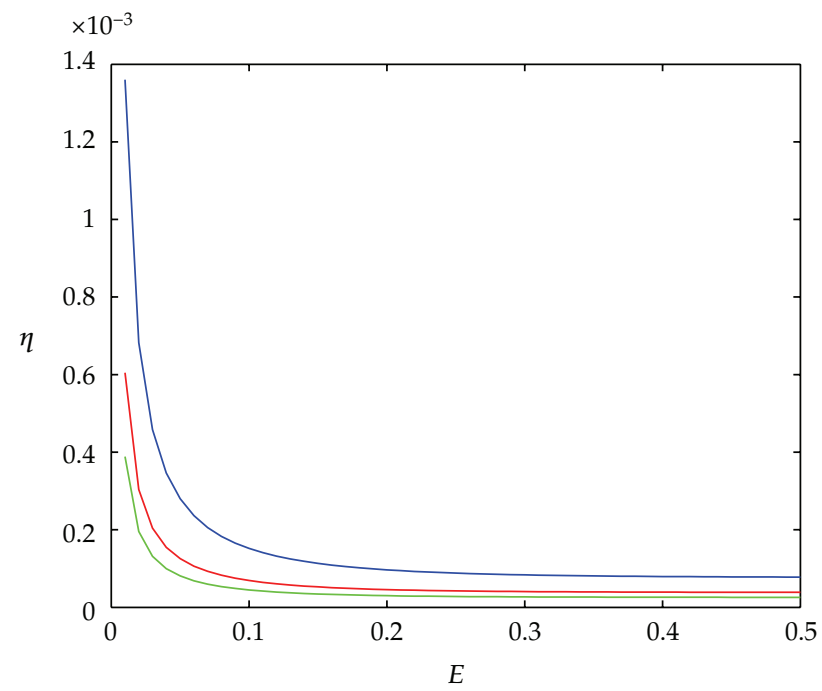

(a) Fin efficiency

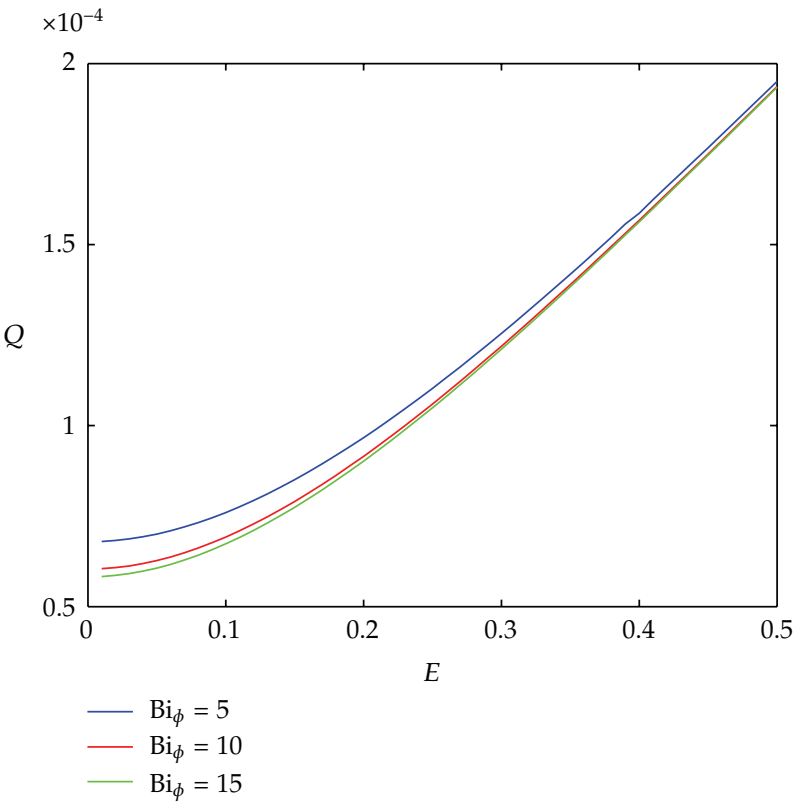

(b) Heat flux

Figure 4: Graphical representation of the heat flux and the fin performance in (5.5) and (5.6), respectively. Here, the first twenty terms of the series in (3.11) are plotted. $\mathrm{Bi}_{\psi}$ is fixed at 0.2 , and $\mathrm{Bi}_{\phi}$ varies as indicated in the graph.

Fin efficiency (5.6) and heat flux (5.5) are depicted in Figures 4(a) and 4(b), respectively. Wherein both heat flux and fin efficiency are plotted against the extension factor. Here, $\mathrm{Bi}_{\psi}$ is fixed at 0.2. In Figure 4(a), we observe that fin performance decreases with increased extension factor. Moreover, the increased Biot number $\mathrm{Bi}_{\psi}$ yields decreased fin efficiency. In our entire analysis, we have assumed a nonuniform internal heat generation. 
Internal heat generation function is assumed to be a uniform in [26]. In Figure 4(b), we observe that Biot number $\mathrm{Bi}_{\psi}$ is inversely proportional to the heat flux. However, heat flux is directly proportional to the extension factor (implying that longer fins result in higher heat flux (see also [14])). The observations in Figures 4(a) and 4(b) are consistent with the results in the literature (see, e.g., [26, 27] or Chapter 15 in [1]). In [26], finite elements methods were used to determine, among others, the effects of uniform internal heat generation. Here, internal energy generation is given as linear function of temperature, and base temperature is quadratic in $y$. Sikka and Iqbal [27] provided the fin efficiency for two-dimensional pin fin. The observations in Figure 4(b) are consistent with those of [27].

\title{
6. Concluding Remarks
}

We have successfully applied the Kirchoff's transformation to reduce the nonlinearity of (2.4) (the source term remained arbitrary). Exact solutions for two-dimensional rectangular fin with temperature-dependent thermal conductivity and heat transfer coefficient, furthermore with internal energy generation function, which depend linearly on temperature are constructed. In the analysis, we allowed the temperature of the fin base to be quadratic in $y$. The forms of the internal energy generation term for which extra symmetries are admitted were obtained. Reduction of the single PDE (given nonlinear source term) to the ODE is achieved. As far as we know, symmetry methods have not yet been employed to two-dimensional fin problems. However, the entire BVP is not invariant under the admitted symmetries. Constructed exact solutions have provided insight into the heat transfer processes in a rectangular straight fin and may be used as benchmarks for the numerical schemes, particularly when thermal conductivity, heat transfer coefficient, and internal thermal energy generation function are all temperature dependent.

\section{Nomenclature}

\author{
$T$ : Temperature distribution \\ $T_{b}$ : Fin base temperature \\ $T_{\infty}$ : Surrounding temperature \\ $\theta: \quad$ Dimensionless temperature \\ $\omega$ : Transformed temperature variable \\ $K: \quad$ Thermal conductivity \\ $K_{a}$ : Thermal conductivity of the fin at the ambient temperature \\ $k$ : $\quad$ Dimensionless thermal conductivity \\ $H$ : Heat transfer coefficient \\ $h_{b}$ : Heat transfer coefficient at the fin base \\ $h$ : Dimensionless heat transfer coefficient \\ $y_{1}:$ Transverse coordinate \\ $x_{1}$ : Longitudinal coordinate \\ $x$ : Spatial variable \\ $y: \quad$ Spatial variable \\ $L: \quad$ Length of the fin \\ l/2: Half width \\ $s(T)$ : Internal energy generation function \\ $S(\theta)$ : Dimensionless internal energy generation function
}




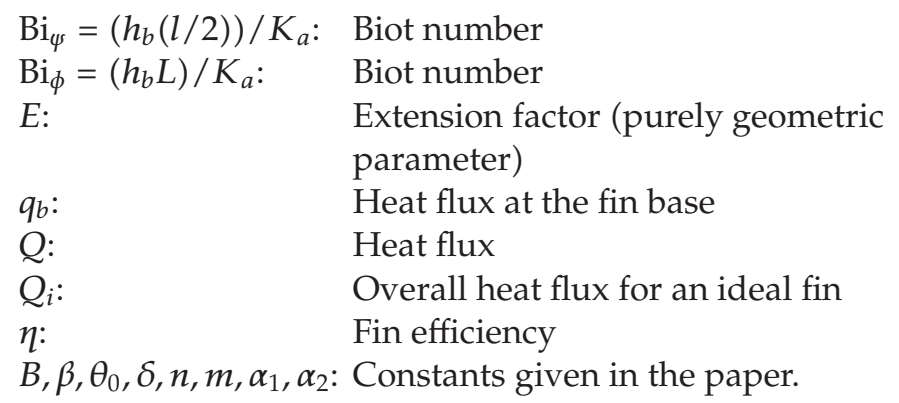

\section{References}

[1] A. D. Kraus, A. Aziz, and J. Welty, Extended Surface Heat Transfer, John Wiley \& Sons, New York, NY, USA, 2001.

[2] B. Sunden and P. J. Heggs, Recent Advances in Analysis of Heat Transfer for Fin Type Surfaces, WIT Press, Boston, Mass, USA, 2000.

[3] F. Khani, M. A. Raji, and H. H. Nejad, "Analytical solutions and efficiency of the nonlinear fin problem with temperature-dependent thermal conductivity and heat transfer coefficient," Communications in Nonlinear Science and Numerical Simulation, vol. 14, no. 8, pp. 3327-3338, 2009.

[4] F. Khani, M. Ahmadzadeh Raji, and S. Hamedi Nezhad, "A series solution of the fin problem with a temeprature-dependent thermal conductivity," Communications in Nonlinear Science and Numerical Simulation, vol. 14, pp. 3007-3017, 2009.

[5] R. J. Moitsheki, T. Hayat, and M. Y. Malik, "Some exact solutions of the fin problem with a power law temperature-dependent thermal conductivity," Nonlinear Analysis: Real World Applications, vol. 11, no. 5, pp. 3287-3294, 2010.

[6] I. N. Dul'kin and G. I. Garas'ko, "Analysis of the 1-D heat conduction problem for a single fin with temperature dependent heat transfer coefficient-part I: extended inverse and direct solutions," International Journal of Heat and Mass Transfer, vol. 51, no. 13-14, pp. 3309-3324, 2008.

[7] R. J. Moitsheki, "Steady heat transfer through a radial fin with rectangular and hyperbolic profiles," Nonlinear Analysis: Real World Applications, vol. 12, no. 2, pp. 867-874, 2011.

[8] E. M. A. Mokheimer, "Heat transfer from extended surfaces subject to variable heat transfer coefficient," Heat and Mass Transfer, vol. 39, no. 2, pp. 131-138, 2003.

[9] M. Y. Malik and A. Rafiq, "Two-dimensional fin with convective base condition," Nonlinear Analysis: Real World Applications, vol. 11, no. 1, pp. 147-154, 2010.

[10] Y. Xia and A. M. Jacobi, "An exact solution to steady heat conduction in a two-dimensional slab on a one-dimensional fin: application to frosted heat exchangers," International Journal of Heat and Mass Transfer, vol. 47, no. 14-16, pp. 3317-3326, 2004.

[11] H. S. Kang and D. C. Look Jr., "Two dimensional trapezoidal fins analysis," Computational Mechanics, vol. 19, no. 3, pp. 247-250, 1997.

[12] A. Aziz and H. Nguyen, "Two-dimensional performance of convecting-radiating fins of different profile shapes," Wärme und Stoffübertragung, vol. 28, no. 8, pp. 481-487, 1993.

[13] L. T. Chen, "Two-dimensional fin efficiency with combined heat and mass transfer between waterwetted fin surface and moving moist airstream," International Journal of Heat and Fluid Flow, vol. 12, no. 1, pp. 71-76, 1991.

[14] A. Aziz and O. D. Makinde, "Heat transfer and entropy generation in a two-dimensional orthotropic convection pin fin," International Journal of Exergy, vol. 7, no. 5, pp. 579-592, 2010.

[15] S. M. Zubair, A. F. M. Arif, and M. H. Sharqawy, "Thermal analysis and optimization of orthotropic pin fins: a closed-form analytical solution," Journal of Heat Transfer, vol. 132, no. 3, pp. 1-8, 2010.

[16] S. Jahangeer, M. K. Ramis, and G. Jilani, "Conjugate heat transfer analysis of a heat generating vertical plate," International Journal of Heat and Mass Transfer, vol. 50, no. 1-2, pp. 85-93, 2007.

[17] M. K. Ranis, G. Jilani, and S. Jahangeer, "Conjugate conduction-forced convection heat transfer analysis of a rectangular nuclear fuel element with nonuniform volumteric energy generation," International Journal of Heat and Mass Transfer, vol. 51, pp. 517-525, 2008.

[18] N. Onur, "A simplified approach to the transient conduction in a two-dimensional fin," International Communications in Heat and Mass Transfer, vol. 23, no. 2, pp. 225-238, 1996. 
[19] D. C. Look Jr., “Two-dimensional fin with non-constant root temperature," International Journal of Heat and Mass Transfer, vol. 32, no. 5, pp. 977-980, 1989.

[20] S. W. Ma, A. I. Behbahani, and Y. G. Tsuei, "Two-dimensional rectangular fin with variable heat transfer coefficient," International Journal of Heat and Mass Transfer, vol. 34, no. 1, pp. 79-85, 1991.

[21] R. M. Cotta and R. Ramos, "Integral transform in the two-dimensional non-linear formulation of longitudinal fins with variable profile," International Journal of Heat and Fluid Flow, vol. 8, no. 1, pp. 27-42, 1998

[22] P. Malekzadeh and H. Rahideh, "Two-dimensional nonlinear transient heat transfer analysis of variable section pin fins," Energy Conversion and Management, vol. 50, pp. 916-922, 2009.

[23] P. J. Olver, Applications of Lie Groups to Differential Equations, Springer, New York, NY, USA, 1986.

[24] G. W. Bluman and S. Kumei, Symmetries and Differential Equations, Springer, New York, NY, USA, 1989.

[25] B. Kundu and P. K. Das, "Performance and optimum designs analysis of convective fin arrays attached to flat and curved primary surfaces," International Journal of Refrigeration, vol. 32, pp. 430-443, 2009.

[26] A. Aziz, "The effects of internal heat generation, anisotropy and base temperature nonuniformity from a two-dimesional rectangular fin," Heat Transfer Engineering, vol. 14, pp. 63-70, 1993.

[27] S. Sikka and M. Iqbal, "Temperature distrubution and effectiveness of a two-dimensional radiating and convecting circular fin," AIAA Journal, vol. 8, no. 1, pp. 101-106, 1970. 


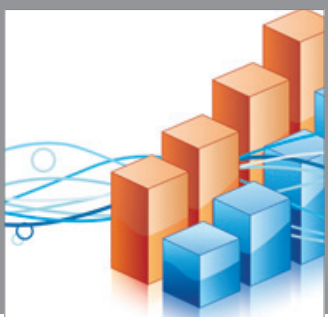

Advances in

Operations Research

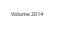

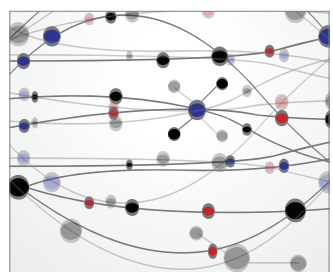

\section{The Scientific} World Journal
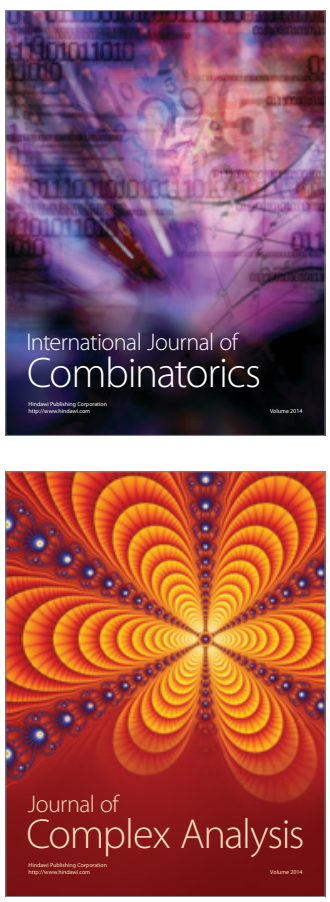

International Journal of

Mathematics and

Mathematical

Sciences
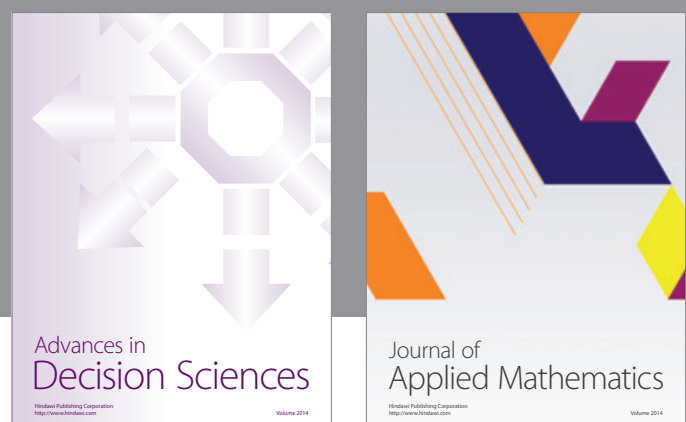

Journal of

Applied Mathematics
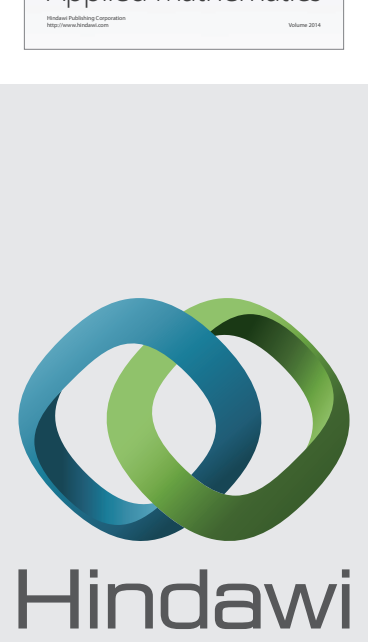

Submit your manuscripts at http://www.hindawi.com
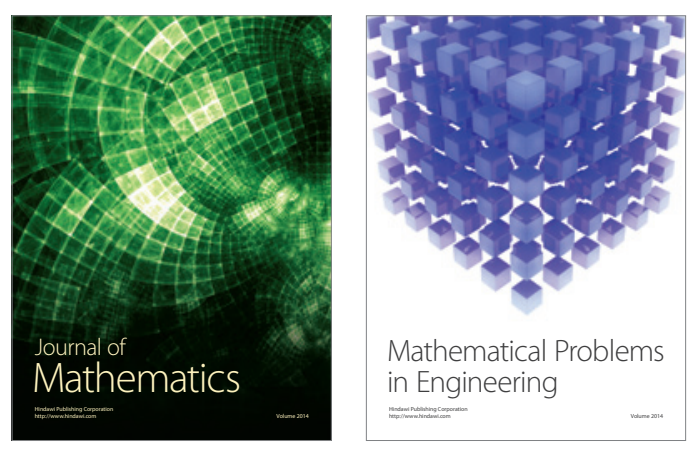

Mathematical Problems in Engineering
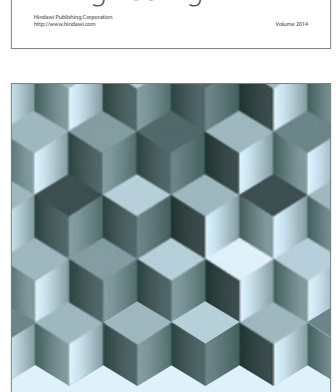

Journal of

Function Spaces
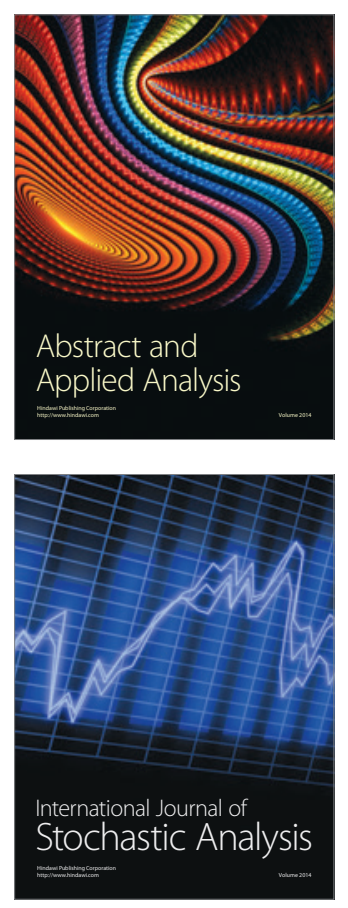

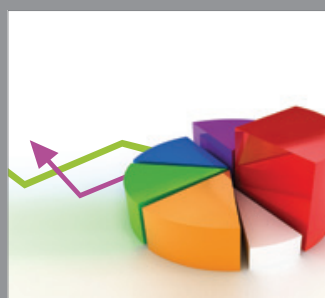

ournal of

Probability and Statistics

Promensencen
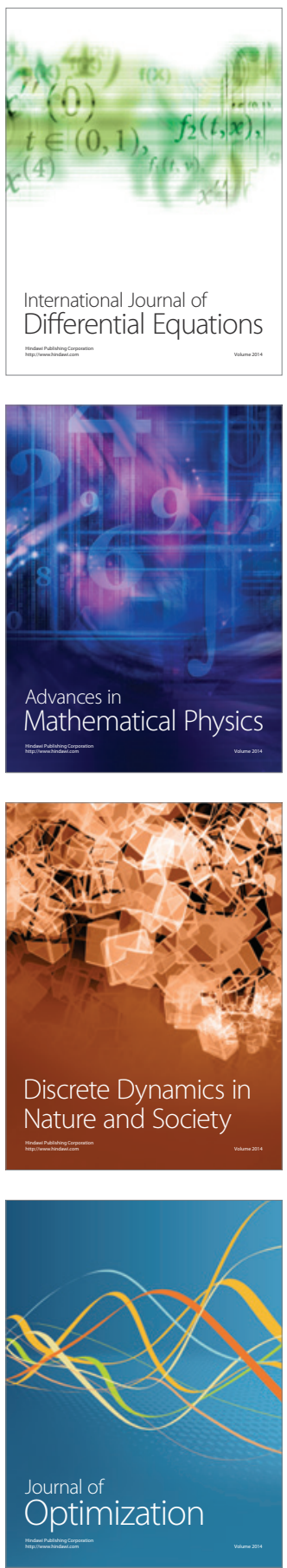